\title{
Photoluminescent SiC nanoparticles synthesized by laser ablation in ethanol medium
}

\author{
E. P. Shuaib, Gaurav Kumar Yogesh and D. Sastikumar*
}

Department of Physics, National Institute of Technology, Tiruchirappalli, Tamilnadu-620015, India

*Email: sasti@nitt.edu

Silicon Carbide (SiC) is a semiconductor material, which has been given much attention due to thewide band gap, high hardness, high thermal conductivity, and good chemical stability. Nano-sized $\mathrm{SiC}$ particle shows photoluminescent properties due to quantum confinement effects which are not observed in bulk counterpart [1]. Commercially available $\mathrm{SiC}$ powders (200-450 mesh) are mixed in ethanol taken in a beaker. The solution shows a greyish coloured solution, which gets easily deposited at the bottom. Laser ablation of the $\mathrm{SiC}$ colloidal solution was performed using a Q-Switched Nd:YAG laser system (QuantaRay) emitting at $1064 \mathrm{~nm}, 10 \mathrm{~Hz}$ having energy $500 \mathrm{~mJ}$ per pulse and $9.5 \mathrm{~mm}$ beam diameter in pulsed mode. Schematic of the laser ablation process is shown in Figure 1. A brownish transparent solution was obtained after 15 minutes of laser irradiation and microparticles were deposited at the bottom of the beaker. The transparent solution containing $\mathrm{SiC}$ nanoparticles have been taken for further analysis and the solution is found to be more stable. The SEM image shows nanoparticle size is below $60 \mathrm{~nm}$.

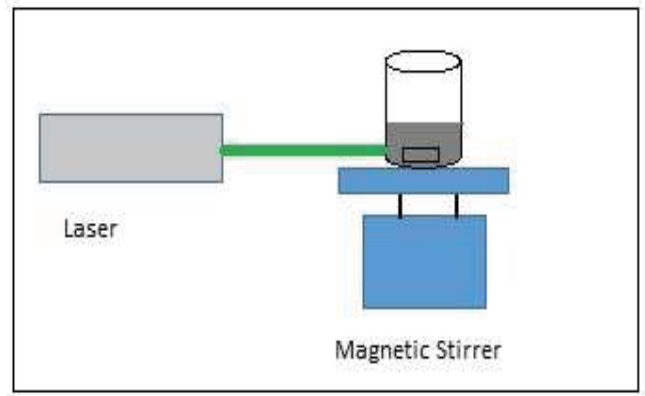

Figure 1: Schematic sketch of the laser ablation of liquid containing $\mathrm{SiC}$ powder

As shown in figure 2, photoluminescence (PL) emission spectrum of $\mathrm{SiC}$ NPs exhibits emission peaks at $409 \mathrm{~nm}$ and $432 \mathrm{~nm}$ for an excitation wavelength of $250 \mathrm{~nm}$, which belongs to the violet region of the visible spectrum. The band gap energy of bulk $\mathrm{SiC}$ is $2.2 \mathrm{eV}$ [2]. However, the band gap of synthesised $\mathrm{SiC}$ nanoparticle from the PL emission is found to be $3.04 \mathrm{eV}$ and $2.87 \mathrm{eV}$, which is much higher than the bulk material. It may be due to the quantum confinement effects.

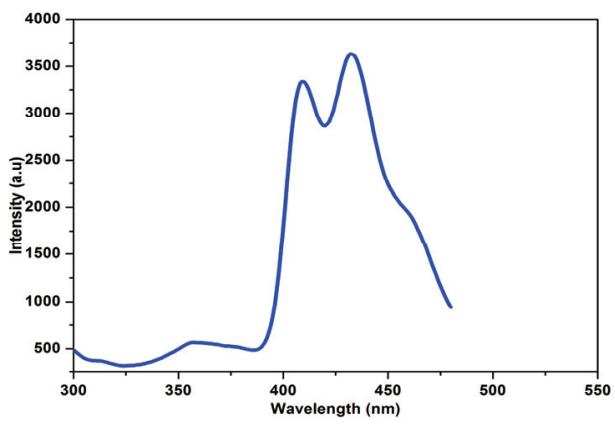

Figure 2: Photoluminescence emission spectra of $\mathrm{SiC}$ NPs corresponding to the excitation wavelength of $250 \mathrm{~nm}$

\section{References}

1. J. Y. Fan, X. L. Wu, H. X. Li, H. W. Liu, G. G Siu, Appl. Phys. Lett.4 (2006) 88

2. W.J. Choyke, L. Patrick, Phys. Rev. B 2 (1970) 4959. 